\title{
Effects of Meditation on Temporal Processing and Speech Perceptual Skills in Younger and Older Adults
}

\author{
Uppunda Ajith Kumar, ${ }^{1}$ A. V. Sangamanatha, ${ }^{2}$ and Jai Vikas ${ }^{3}$ \\ ${ }^{1}$ Department of Audiology, All India Institute of Speech and Hearing, Manasagangotri, Mysore, India \\ ${ }^{2}$ National Center for Audiology, Elborn College, University of Western Ontario, London, ON, Canada N6G $1 H 1$ \\ ${ }^{3}$ National Hearing Care, Suite 2, Northbourne Avenue, Canberra, ACT, Australia
}

Correspondence should be addressed to A. V. Sangamanatha; sangamanatha@gmail.com

Received 12 June 2013; Accepted 17 July 2013

Academic Editors: M.-c. Cheung and M. L. Seghier

Copyright (C) 2013 Uppunda Ajith Kumar et al. This is an open access article distributed under the Creative Commons Attribution License, which permits unrestricted use, distribution, and reproduction in any medium, provided the original work is properly cited.

\begin{abstract}
The purpose of this study was to assess the temporal processing and speech perception abilities in older adults who were practicing meditation for more than five years. Participants were comprised of three groups, 30 young adults ("YA") in the age range of 20-30 years, 30 older adults in the age range of 50-65 years who practiced meditation for a period of five years or more (effective meditators "EM"), and 51 age matched older adults who did not have any experience of meditation (non-meditators "NM"). Temporal processing was evaluated using gap detection in noise, duration discrimination, modulation detection, and backward masking and duration pattern tests. Speech perception was measured in presence of a four-talker babble at $-5 \mathrm{~dB}$ signal to noise ratio and with the vocoded stimuli. Results revealed that EM group performed significantly better than NM group in all psychophysical and speech perception tasks except in gap detection task. In the gap detection task, two groups did not differ significantly. Furthermore, EM group showed significantly better modulation detection thresholds compared to YA. Results of the study demonstrate that the practice of meditation not only offsets the decline in temporal and speech processing abilities due to aging process but also improves the ability to perceive the modulations compared to young adults.
\end{abstract}

\section{Introduction}

Auditory temporal processing is the perception of sound or of the alteration of sound within a restricted or defined time domain [1]. Speech stimuli and other background sounds are dynamic in terms of both amplitude and frequency. Perception of this variation in amplitude and frequency is crucial to understand speech in quiet and more so in background noise [2]. Psychophysical evidence indicates deterioration in a broad spectrum of auditory abilities as a result of chronological aging [3]. Thus, the deterioration in temporal processing may also be expected with the aging. Evidence for deterioration of temporal processing with age predominantly comes from studies on gap detection. Investigators have shown increased gap detection thresholds in elderly population [4-7]. Some investigators have used simple tonal or noise signals and reported age-related difficulties in detection of gap $[6,8]$, whereas others have used more complex stimuli or increased task demands and shown more pronounced differences between younger and older listeners [9]. Additionally, older individuals are also shown to have difficulties with auditory sequencing tasks that require discrimination or recognition of temporal order of the stimulus within a serial pattern [10-13].

Supporting evidence for the decline in temporal processing with the age also comes from speech perception studies using complex and acoustically degraded speech stimulus. It has been reported that older listeners experience increased difficulty in understanding speech in noise (e.g., $[14,15])$. Presence of noise reduces the temporal variation of the waveform by filling the valleys of the amplitude spectrum. These amplitude variations in the temporal envelope of the speech signal and periodicity have been shown to convey important information about syllable and phrase boundaries, 
voicing, and consonant identification [16, 17]. Degradation of temporal envelope may distort the speech signal and consequently reduce the speech understanding. Peripheral hearing loss and cognitive decline which accompany aging will induce additional difficulties in understanding speech. Furthermore, it has also been suggested that older listeners are more susceptible to the irrelevant or distractive stimuli because of age-related changes in cognitive functioning, especially the working memory capacity. Working memory is important for auditory perceptions of both speech and nonspeech stimuli [18]. Speech recognition decline associated with peripheral hearing loss can be rehabilitated with amplification devices that provide audibility of signals. Speech recognition declines associated with poor temporal processing or poor cognitive mechanism may not necessarily be helped by amplification.

Meditation practices have various health benefits including possibility of preserving cognition and preventing brain aging. It has been shown that both the short-term and longterm meditation improves the attentional resources $[19,20]$. Slagter et al. [19] reported that three months of intensive meditation training resulted in improvement in executive attentional network, as measured by attentional blink test. Tang et al. [20] reported that even short-term meditation training for 5 days significantly improved the attention and reduced the stress. Furthermore, meditation is also reported to improve the other cognitive faculties such as working memory capacities and some executive functions. Physiologically it has been shown that meditation affects the lipid profiles and lowers the oxidative stress. This can reduce the age-related neurodegeneration [21]. Neurophysiological lines evidence suggest that meditation may potentially strengthen neuronal circuits and enhance cognitive and/or sensory reserve capacity. Evidence to this claim comes from electroencephalogram (EEG)/evoked potential (for review see [22]), structural [23], and functional neuroimaging studies [24]. EEG studies have demonstrated increase in alpha and theta power and decrease in over all frequency distribution in some individuals when meditating compared with the baseline EEG (for review see [25]). Lazar et al. [23] reported increased thickness in cortical areas associated with attention, introspection, and sensory processing in meditation participants compared to those in the matched controls. Increased thicknesses in the prefrontal cortical areas were most pronounced in older participants practicing meditation, suggesting that meditation might offset the age-related cortical thinning. Baron-Short et al. [26] reported increased activation in brain regions associated with attention such as dorsal lateral prefrontal cortex and anterior cingulate cortex during meditation. Srinivasan and Baijal [27] reported larger amplitudes of auditory evoked mismatch negativity in individuals who practiced meditation compared to those in the control group.

We hypothesized that, as suggested by behavioral and physiological studies, if meditation results in reorganization of neuronal circuitry and improves cognitive abilities such as selective and executive attention and working memory then this beneficial effect should be reflected in the auditory domain too. In this study, we evaluated the speech recognition and temporal processing abilities of older adult individuals who were practicing meditation for more than
TABLE 1: Mean and standard deviations of MMSE scores.

\begin{tabular}{lcc}
\hline Group & Mean & Standard deviation \\
\hline Young adults & 29.4 & 0.81 \\
Effective meditators & 29.5 & 0.76 \\
Nonmeditators & 29.3 & 0.86 \\
\hline
\end{tabular}

five years. Results were compared with matched older adults group and with young adults.

\section{Method}

2.1. Participants. A total of 111 participants participated in this research. The participants were comprised of three groups, 30 young adults (hereafter called "YA") in the age range of 20-30 years (mean age: 20.2 years, 15 males), 30 older adults in the age range of 50-65 years (mean age: 56.88 years, 20 males) who practiced meditation for a period of five years or more (hereafter called "EM" (effective meditators)), and 51 age matched older adults (mean age: 57.9 years, 40 males) who did not have any experience of meditation (hear after called "NM" (nonmeditators)). All the members of EM group had learnt the meditation from a qualified teacher and were practicing "Sahaj Marg Meditation" (a raja yoga system of spiritual training based on heart centered system of meditation). All of them were practicing meditation for at least two hours a day for a period of five years. Participants in all the three groups had hearing thresholds within $15 \mathrm{~dB} \mathrm{HL}$ in the octave frequencies between $250 \mathrm{~Hz}$ and $8 \mathrm{kHz}$. None of the participants reported any otologic or neurological problems. All the participants were native speakers of Kannada, a Dravidian language. Mini-Mental Status Examination (MMSE) was administered on all the participants to rule out any gross cognitive deficits. Table 1 shows the mean and standard deviation of MMSE scores. MMSE scores were within normal limits for all three groups. ANOVA did not show any significant effect on MMSE scores $(F(2,108)=0.12, P>0.05)$. Table 2 shows the details of the meditation practice by the EM group.

\subsection{Stimulus and Procedure}

2.2.1. Psychophysical Tests. All temporal processing tests except for the duration pattern test were carried out using "maximum likelihood procedure" tool box which implements a maximum likelihood procedure in Matlab [28]. The maximum likelihood procedure employs a large number of candidate psychometric functions and after each trial calculates the probability (or likelihood) of obtaining the listener's response to all of the stimuli that have been presented given each psychometric function. The psychometric function yielding the highest probability is used to determine the stimulus to be presented on the next trial. Within about 12 trials, the maximum likelihood procedure usually converges on a reasonably stable estimate of the most likely psychometric function, which then can be used to estimate threshold $[29,30]$. Stimuli were reproduced at $44,100 \mathrm{~Hz}$ sampling rate. A twointerval alternate forced choice method using a "maximum 
TABLE 2: Details of the meditation practice by EM group.

\begin{tabular}{|c|c|c|}
\hline Participants & $\begin{array}{l}\text { Number of years of } \\
\text { meditation practice }\end{array}$ & $\begin{array}{l}\text { Age at which meditation } \\
\text { practice was started }\end{array}$ \\
\hline 1 & 30 & 35 \\
\hline 2 & 10 & 51 \\
\hline 3 & 30 & 35 \\
\hline 4 & 7 & 33 \\
\hline 5 & 5 & 35 \\
\hline 6 & 10 & 37 \\
\hline 7 & 6 & 37 \\
\hline 8 & 8 & 42 \\
\hline 9 & 8 & 44 \\
\hline 10 & 8 & 45 \\
\hline 11 & 9 & 41 \\
\hline 12 & 10 & 60 \\
\hline 13 & 10 & 50 \\
\hline 14 & 5 & 54 \\
\hline 15 & 6 & 54 \\
\hline 16 & 10 & 55 \\
\hline 17 & 10 & 40 \\
\hline 18 & 10 & 40 \\
\hline 19 & 5 & 50 \\
\hline 20 & 6 & 50 \\
\hline 21 & 7 & 46 \\
\hline 22 & 8 & 52 \\
\hline 23 & 5 & 45 \\
\hline 24 & 7 & 45 \\
\hline 25 & 8 & 42 \\
\hline 26 & 10 & 40 \\
\hline 27 & 8 & 47 \\
\hline 28 & 9 & 47 \\
\hline 29 & 8 & 45 \\
\hline 30 & 10 & 50 \\
\hline
\end{tabular}

likelihood procedure" was employed to track an $80 \%$ correct response criterion. During each trial, stimuli were presented in each of two intervals: one interval contained a reference stimulus, the other interval contained the variable stimulus. The participant indicated after each trial which interval contained the variable stimulus. This procedure was used in all temporal processing tests except for the duration pattern test. Stimuli for the latter test were generated using Audacity software 1.3.5 (beta version 2008). In all of the psychophysical tests, stimuli were presented binaurally at an intensity of $80 \mathrm{~dB}$ SPL. Stimuli were presented via a laptop computer (Compaq Presario C700) connected to EAR-3A earphones. Output of the earphones was calibrated at the beginning of the experiment and regularly thereafter to produce $80 \mathrm{~dB}$ SPL for a $1 \mathrm{kHz}$ pure tone in a $2 \mathrm{cc}$ coupler. For this purpose, a $1 \mathrm{kHz}$ pure tone was generated at the same rms level as the test signal. Output of the earphone was routed to a $2 \mathrm{cc}$ coupler which was connected to a sound level meter (Quest 1800) and a microphone (Quest 4180). The volume control of the computer was adjusted to produce $80 \mathrm{SPL}$ on the sound level meter. Participants were given 3-4 practice trials before the commencement of each test. All psychophysical tests were carried out in a quiet room in 2-3 sessions with 5-10 minutes of rest period between each session.

Gap Detection in White Noise. This was measured by asking the participants to detect a temporal gap in the center of $750 \mathrm{~ms}$ band pass noise $(400-1600 \mathrm{~Hz})$. Duration of gap was varied according to the listener performance using maximum likelihood procedure. The noise had $0.5 \mathrm{~ms}$ cosine ramps at the beginning and end of the gap. In two interval alternate force choice tasks, the standard stimulus was always a $750 \mathrm{~ms}$ broadband noise with no gap whereas the variable stimulus contained the gap.

Duration Discrimination. In this, the minimum difference in duration that was necessary to perceive the two otherwise identical $1000 \mathrm{~Hz}$ pure tone was measured. Duration of the standard stimuli was $250 \mathrm{~ms}$. Duration of the variable stimuli was changed according to subject's response. In two intervals of alternate forced choice procedure, subject's task was to tell which interval contained the longer duration signal.

Modulation Detection Thresholds. Temporal modulation refers to a reoccurring change (e.g., frequency or amplitude) in the signal over time. A $500 \mathrm{msec}$ Gaussian noise was sinusoidally amplitude modulated at $8 \mathrm{~Hz}, 20 \mathrm{~Hz}, 60 \mathrm{~Hz}$, and at $200 \mathrm{~Hz}$ modulation frequencies. The subject had to detect the modulation and determine which interval had the modulated noise. Modulated and unmodulated stimuli were equated for total RMS power. Depth of the modulated signal was varied according to participants response to track $80 \%$ criterion level. Noises had two $10 \mathrm{msec}$ raised cosine ramps at onset and offset. The modulation detection thresholds were expressed in $\mathrm{dB}$ using the following equation:

$$
\text { modulation detection thresholds in } \mathrm{dB}=20 \log _{10} m \text {, }
$$

where $m=$ modulation detection threshold in percentage.

Backward Masking. In this procedure, the masker followed the signal with no time interval between two. A $20 \mathrm{~ms}, 1 \mathrm{kHz}$ pure tone (the signal) was presented immediately before (i.e., no silent gap) band pass noise of $300 \mathrm{~ms}(400-1600 \mathrm{~Hz})$. Both noise and tone had rise and fall time of $10 \mathrm{~ms}$. The participants' task was to tell which interval had the tone. Intensity of the pure tone was changed depending on subject's response to track the backward masked thresholds.

Duration Pattern Test. Duration pattern test was administered in the manner described by Musiek et al. [31]. A $1000 \mathrm{~Hz}$ pure tone was generated with two different durations (i.e., short $250 \mathrm{~ms}$ and long $500 \mathrm{~ms}$ ). By combining these two durations in a three-tone pattern, six different patterns were generated (Short Short Long, Short Long Short, Long Long Short, Long Short Short, Short Long Long, Long Short Long). Participants were asked to repeat the order of tones verbally. Following practice trails, the 30 test items were administered. 
Participants were asked to verbally repeat the responses. The order of psychophysical tests was counterbalanced between participants to avoid the order effect if any.

\subsubsection{Speech Perception Tests}

Speech Recognition with Multitalker Babble. Speech recognition was tested using custom made sentence material. Material consisted of 10 sentences, each containing 4-5 key words. There were a total of 44 key words. A four-talker babble was added to the sentences at $-5 \mathrm{~dB}$ SNR using a custom written Matlab code. The program first calculated the root mean square amplitude of speech stimuli and then adjusted the root mean square amplitude of babble to achieve desired signal to noise ratio. These sentences were randomly presented binaurally using a personal computer at an intensity of $70 \mathrm{~dB}$ SPL. Output of the computer was calibrated in the beginning of the experiment and then regularly in between as described in the previous section. Participants were asked to repeat the sentences. Subject's responses were voice recorded for further analysis. Each of the correctly repeated key words was given a score of "1", and the total number of correct responses was calculated for individual participants. These scores were arcsine-transferred for further analysis.

Speech Recognition with Vocoded Stimuli. Same sentences that were used for speech recognition in multitalker babble were used for measuring speech recognition with vocoded stimuli. This kind of the degradation removes all the spectral content from the speech, leaving only the temporal (envelope) cues, and gives an estimate of individuals capability to understand speech only with temporal cues. This represents the approximation of auditory stimulus received by cochlea implant users. Envelopes of the sentences were extracted with the help of frequency amplitude modulation encoding [32] algorithm. Briefly, stimulus was first filtered into 16 logarithmically spaced filters spanning a frequency range of 80-8000 Hz [33]. The band-limited signal was then decomposed by the Hilbert transform into a slowly varying temporal envelope and a relatively fast-varying fine structure. The number of bandlimited filters was chosen to avoid cochlear filtering with a low number of filters and filter ringing with a high number of filters [34]. The new stimuli with primarily temporal envelope cue were created by modulating each filters' center frequency by slowly varying temporal envelope and then summing the modulated subband signals. The procedure used for presentation of the stimuli and collection of responses was the same as that used for speech recognition with multitalker babble. The order of testing was counterbalanced between participants to avoid order effects. Scoring and analysis procedure was similar to speech recognition with multitalker babble.

\section{Results}

3.1. Psychophysical Tests. Figure 1 represents the means and one-standard-deviation error bars of three participant groups for gap detection thresholds, duration discrimination thresholds, backward masking thresholds, and duration pattern scores. Figure 2 shows the temporal modulation transfer
TABLE 3: $F$ values and degrees of freedom obtained on univariate ANOVA.

\begin{tabular}{|c|c|c|}
\hline Psychophysical test & Degrees of freedom & $F$ value \\
\hline Gap detection in noise & 2,110 & $38.1^{* *}$ \\
\hline Duration discrimination thresholds & 2,110 & $34.7^{* *}$ \\
\hline $\begin{array}{l}\text { Modulation detection thresholds at } \\
8 \mathrm{~Hz}\end{array}$ & 2,110 & $20.03^{* *}$ \\
\hline $\begin{array}{l}\text { Modulation detection thresholds at } \\
20 \mathrm{~Hz}\end{array}$ & 2,110 & $14.6^{* *}$ \\
\hline $\begin{array}{l}\text { Modulation detection thresholds at } \\
60 \mathrm{~Hz}\end{array}$ & 2,110 & $50.4^{* *}$ \\
\hline $\begin{array}{l}\text { Modulation detection thresholds at } \\
200 \mathrm{~Hz}\end{array}$ & 2,110 & $17.8^{* *}$ \\
\hline Backward masked thresholds & 2,110 & $15.8^{* *}$ \\
\hline Duration pattern scores & 2,110 & $20.9^{* *}$ \\
\hline
\end{tabular}

${ }^{* *} P<0.01$.

function for three groups along with one-standard-deviation error bars. A multivariate analysis of variance (MANOVA) was conducted to find significance of differences between the means of psychophysical test scores among the three groups. MANOVA revealed a significant main effect of subject groups $(F(16,202)=18.721, P<0.01)$. Follow-up univariate ANOVAs indicated that subject groups had a significant effect on all the five psychophysical measures. Table 3 shows the $F$ values and degrees of freedom of univariate ANOVAs. Bonferroni's post hoc comparisons were done to evaluate the effect of meditation and age on each psychophysical measure separately. The results of post hoc comparisons were as follows.

(a) YA group performed significantly better than NM group on all the temporal processing tests.

(b) EM group performed significantly better than NM group on duration discrimination, duration pattern, and backward masking measures.

(c) There was no significant difference between EM and NM group in gap detection thresholds.

(d) EM group showed significantly better modulation detection thresholds compared to both YA and NM group.

These results in combination suggest that performance of individuals who practice meditation was on par with that of young adults.

3.2. Speech Perception Tests. The mean percent correct identification scores (and one standard deviation) in multitalker babble and vocoded condition for three subject groups are shown in Figure 3. Prior to conducting statistical analysis, the percent correct scores were transformed using rationalized arcsine transformation [35]. MANOVA showed significant main effect of subject group on speech recognition scores $(F(2,108)=140.317, P<0.00)$. Bonferroni's post hoc comparisons showed that NM group had significantly poorer speech recognition scores compared to EM and YA groups 


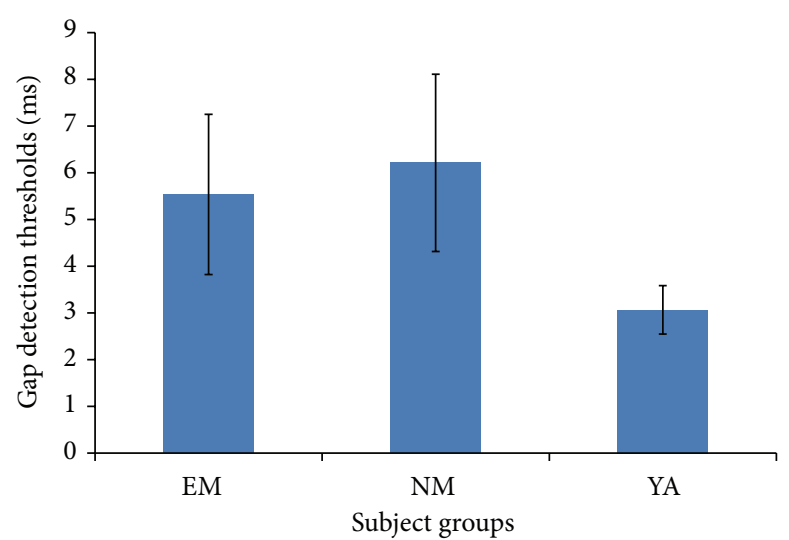

(a)

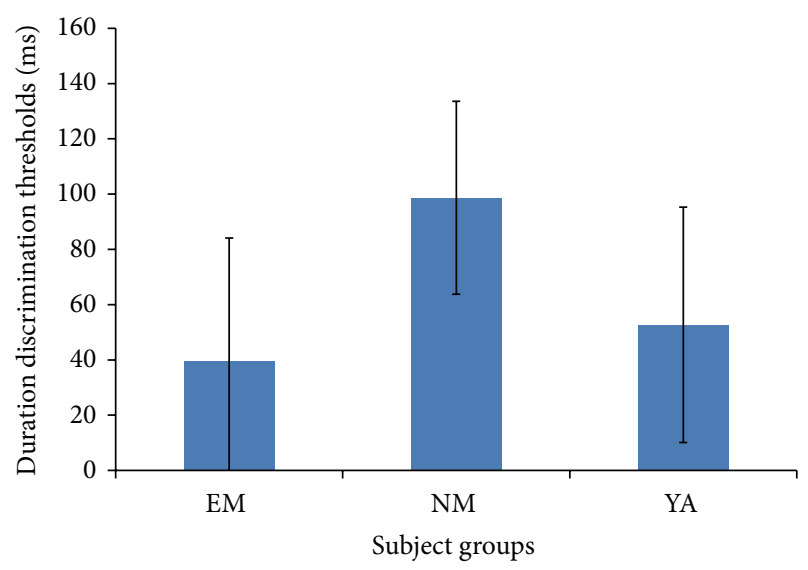

(c)

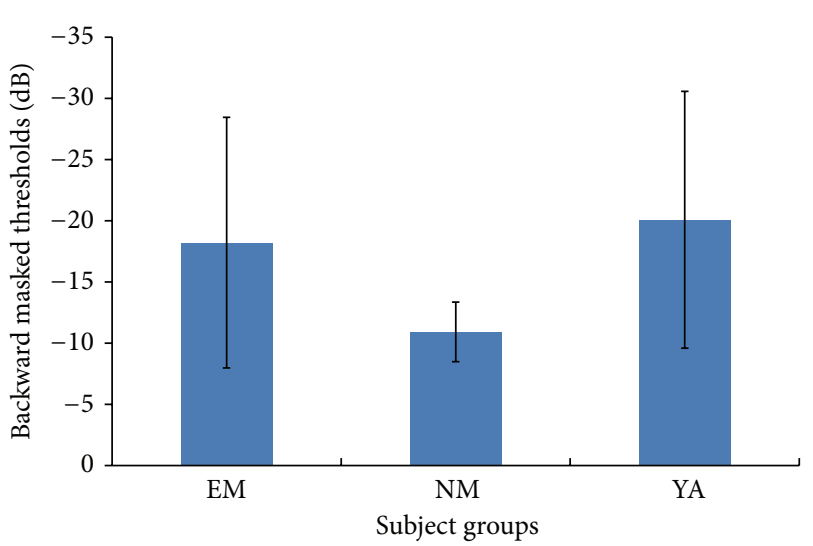

(b)

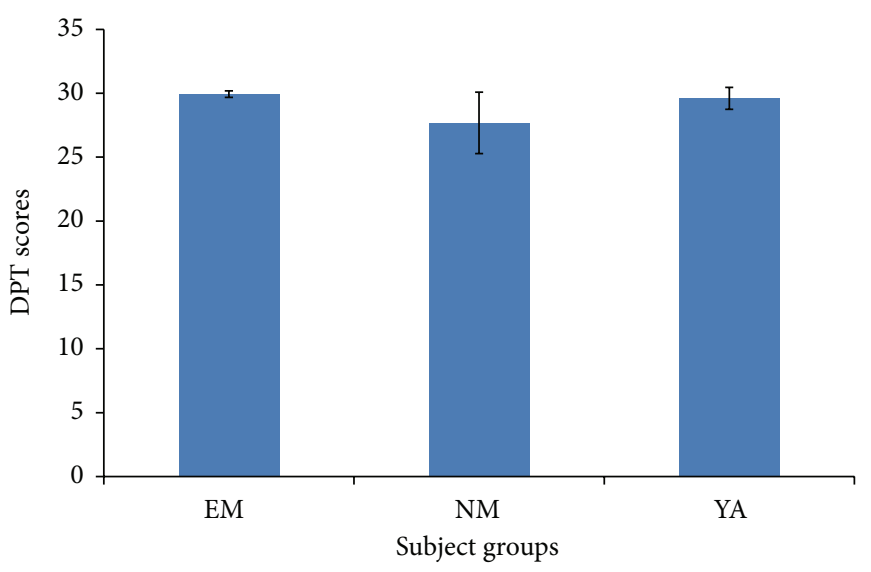

(d)

FIGURE 1: Mean and one-standard-deviation error bars of three participant groups for (a) gap detection thresholds, (b) backward masking thresholds, (c) duration discrimination thresholds (d) duration pattern scores. EM: effective meditators; NM: nonmeditators; YA: young adults.

for both speech in noise and vocoded sentences. YA and EM groups did not differ in both conditions.

3.3. Relationship between Meditation and Auditory Measures. To evaluate the relationship between meditation and auditory measures, Pearson's product moment correlation coefficients were calculated with different auditory measures as dependent variables and years of meditation practice and age at which meditation practice was started as independent variables. Results failed to reveal any significant relationship among auditory measures and meditation.

\section{Discussion}

The word "meditation" refers to practices that self-regulate the body and mind, thereby affecting the mental events by engaging a specific attentional set [25]. Sahaj Marg, meaning "natural path" or "simple way," is a system of practical training in spirituality and meditation. It is in essence the traditional practice of raja yoga (yoga of the mind), remodelled and simplified to help people achieve inner perfection or God realisation. Previous research has shown that meditation has several

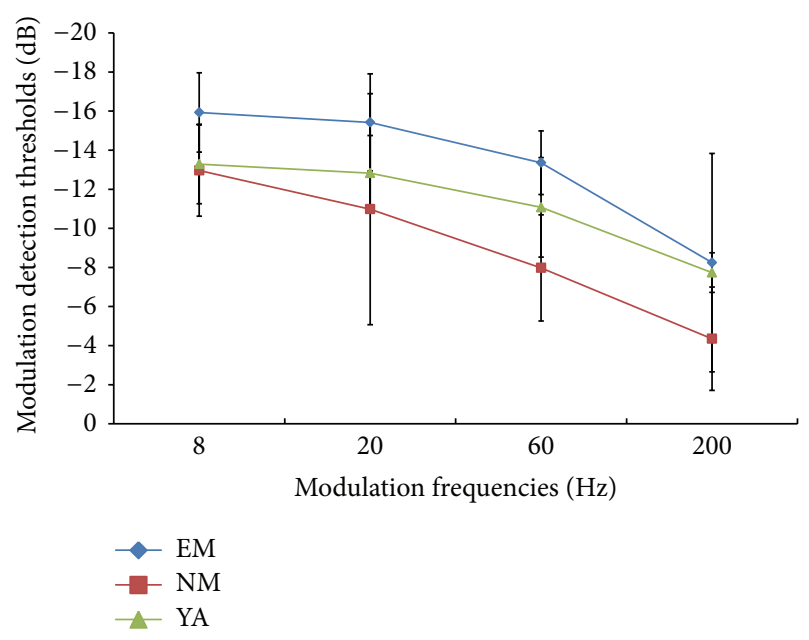

Figure 2: Temporal modulation transfer function in different participant groups. Error bars depict one standard deviation of error. EM: effective meditators; NM: non meditators; YA = young adults.

health benefits including possibility of preserving cognition and preventing brain aging [21]. Much of the evidence for 


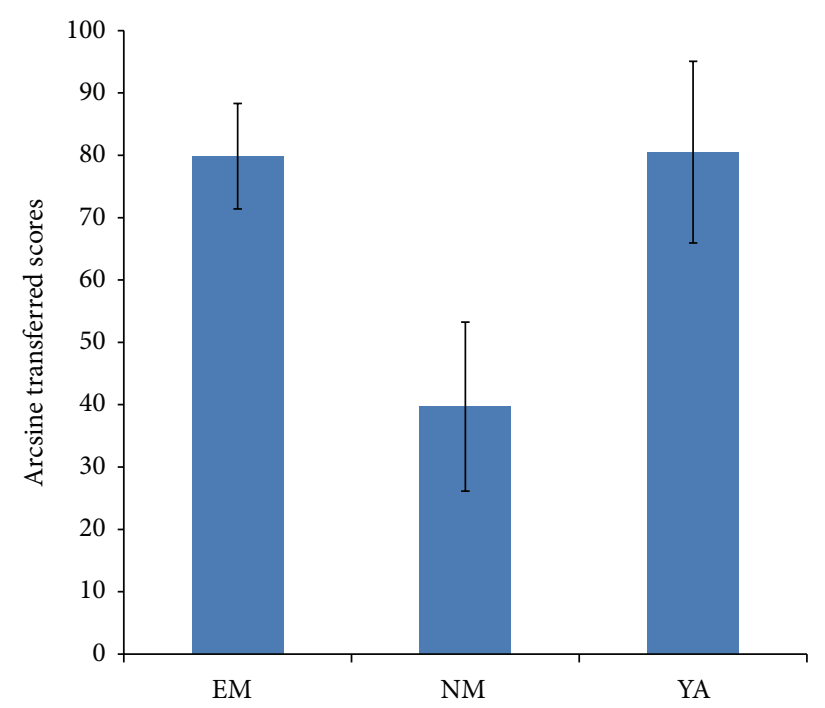

(a)

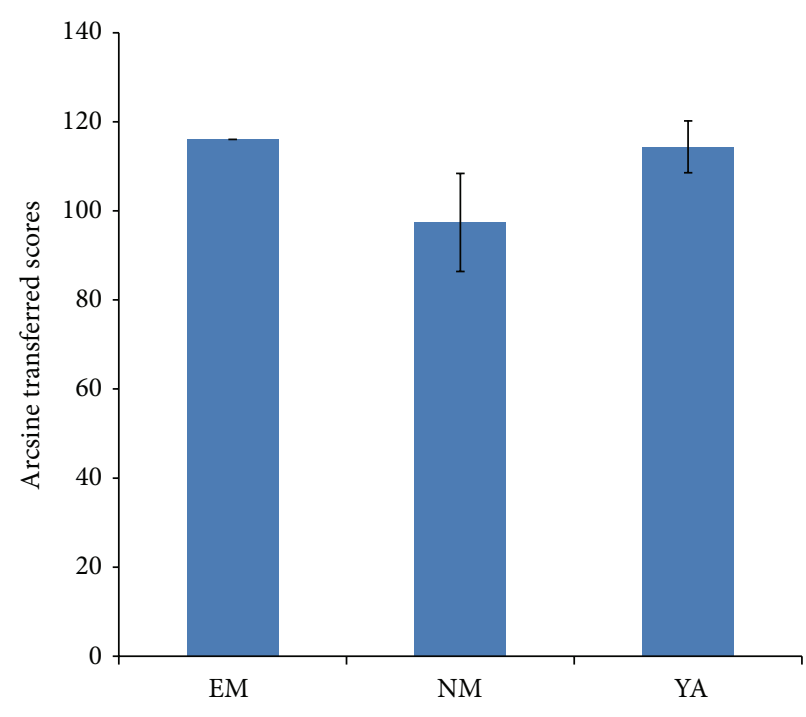

(b)

FIGURE 3: Speech perception scores (a) in presence of $-5 \mathrm{~dB}$ SNR (b) with vocoded sentences three participant groups. Error bars depict one standard deviation of error. EM: effective meditators; NM: non meditators; YA: young adults.

positive effects of meditation comes from electrophysiological and imaging studies of the nervous system. To our knowledge, perhaps this is the first study to use auditory behavioral paradigm, specifically temporal and speech processing skills, to investigate the changes owing to the practice of meditation. Results of the present study indicate that temporal and speech perception abilities of individuals who practice "Sahaj Marg meditation" were superior compared to these of age matched controls. It is also worth noting that elderly control group (NM) performed significantly poorer temporal processing and speech perception skills compared to young adults. These results are consistent with the previous studies which have shown decline in temporal processing and speech perception abilities with the advancing age $[7,14]$. We did not observe any relationship between auditory measures and meditation history. This is probably because other than 2 participants, all had meditation experience between 5 and 10 years and commenced meditation practices after the 3rd decade of life. Lack of relationship between these two variables does not rule out the cause and effect relationship between them. However, it should be kept in mind that we did not control for the musical skills or the music listening habits of the participants. It has been shown that musicians have better auditory skills when compared to nonmusicians [36] and this could have influenced the results of the current study if a particular group had more or less musicians than the other group.

Extracting acoustic cues involves separating (grouping) and organizing the psychoacoustic cues over a period of time [36]. This process is essential for understanding speech in adverse listening condition. Extracting these cues from the background noise draws attentional resources resulting in shorter auditory memory span available for understanding the speech [37]. Individuals with better attention and working memory may spend less time in extracting the acoustic cues in noisy conditions and may spend more time in rehearsal and recall of the target word. Studies have reported reduced working memory span in adverse listening condition [38]. It is well documented that meditation improves the cognitive functioning including working memory capacities and selective and executive attention $[19,20]$. The same cognitive functions play a crucial role even in auditory perception also [39].

Lazar et al. [23] showed that cortical regions associated with somatosensory, visual, and auditory processing were thicker in individuals who practice meditation than those of the age matched controls. The differences in the cortical thickness were most pronounced in older participants, specifically in prefrontal regions, suggesting that meditation might offset age-related cortical thinning. Hölzel et al. [40] noted that meditation practices activated the rostral anterior cingulate gyrus and dorsal medial prefrontal cortex, suggesting better attention regulation in individuals who practice meditation. In accordance with these results, there are some lines of evidence to show that meditators have superior attentional performance especially to unexpected stimuli compared to nonmeditators [41, 42]. Slagter et al. [19] reported that three months of intensive meditation training resulted in improvement in executive attentional network, as measured by attentional blink test. Tang et al [20] reported that even short-term meditation training for 5 days significantly improved the attention and reduced the stress. Furthermore, certain biochemical differences have been reported in individuals who practice meditation compared to nonmeditators [22].

Wong et al. [43] investigated the cortical mechanisms of hearing in noise in elderly participants using fMRI technique. They reported that elderly participants had poor speech recognition abilities in noise (at $-5 \mathrm{~dB}$ SNR) compared to young adults. Elderly individuals showed less activation in auditory areas (bilateral superior temporal gyrus) and more activation in prefrontal and regions precuneus (working 
memory and attentional networks). Furthermore, the activation in the prefrontal and precuneus regions was positively correlated with behavioral performance in noise. These results in combination suggest that elderly individuals require more attention and working memory while listening to speech in noise, and meditation prevents the age-related thinning of cortical areas associated with these functions. Prefrontal regions have also been indicated as a part of dynamic network of cortical-subcortical regions associated with the different components of temporal information processing [44]. This slowing down of age related cortical thinning may be one of the reasons for better performance of meditators on speech and temporal processing tests. On modulation detection tasks, individuals who practice meditation performed even better than young adults. These results show that practice of meditation not only offsets the decline in modulation detection abilities due to aging process but also improves the ability to perceive the modulations compared to young adults.

Recently, in a series of studies, Kraus and her colleagues have demonstrated a number of beneficial effects of music on auditory perception and cognition (e.g., $[36,45])$. They showed that musicians with experience of more than 10 years of music practice had better speech in noise, auditory temporal processing, and auditory working memory skills. Results of the present study indicate that meditation can also have beneficial effects on auditory temporal processing and speech perception in noise. These preliminary results suggest that meditation can be one of the rehabilitative options to offset the age-related decline in auditory and speech processing. It may be one the adjunctive therapeutic techniques to augment the benefits from amplification devices in elderly individuals with hearing impairment. However, more systematic and controlled investigation is required before clinically using meditation as one of the rehabilitative techniques in elderly hearing-impaired individuals.

\section{References}

[1] J. B. Shinn and F. E. Musiek, "Temporal processing: the basics," Hearing Journal, vol. 56, no. 7, p. 52, 2003.

[2] S. Gordon-Salant and P. J. Fitzgibbons, "Temporal factors and speech recognition performance in young and elderly listeners," Journal of Speech and Hearing Research, vol. 36, no. 6, pp. 12761285, 1993.

[3] P. J. Fitzgibbons and S. Gordon-Salant, "Behavioral studies with aging humans: hearing sensitivity and psychoacoustics," in The Aging Auditory System, S. Gordon-Salant, R. D. Frisina, A. N. Popper, and R. R. Fay, Eds., Springer, London, UK, 2010.

[4] D. M. Green, “Temporal auditory acuity," Psychological Review, vol. 78, no. 6, pp. 540-551, 1971.

[5] P. J. Fitzgibbons and F. L. Wightman, "Gap detection in normal and hearing-impaired listeners," The Journal of the Acoustical Society of America, vol. 72, no. 3, pp. 761-765, 1982.

[6] K. B. Snell, "Age-related changes in temporal gap detection," Journal of the Acoustical Society of America, vol. 101, no. 4, pp. 2214-2220, 1997.

[7] U. Ajith Kumar and A. V. Sangamanatha, "Temporal processing abilities across different age groups," Journal of the American Academy of Audiology, vol. 22, no. 1, pp. 5-12, 2011.
[8] B. A. Schneider, M. K. Pichora-Fuller, D. Kowalchuk, and M. Lamb, "Gap detection and the precedence effect in young and old adults," Journal of the Acoustical Society of America, vol. 95, no. 2, pp. 980-991, 1994.

[9] P. J. Fitzgibbons and S. Gordon-Salant, "Age effects on duration discrimination with simple and complex stimuli," Journal of the Acoustical Society of America, vol. 98, no. 6, pp. 3140-3145, 1995.

[10] P. J. Fitzgibbons and S. Gordon-Salant, "Auditory temporal processing in elderly listeners," Journal of the American Academy of Audiology, vol. 7, no. 3, pp. 183-189, 1996.

[11] P. J. Fitzgibbons and S. Gordon-Salant, "Auditory temporal order perception in younger and older adults," Journal of Speech, Language, and Hearing Research, vol. 41, no. 5, pp. 1052-1060, 1998.

[12] L. E. Humes and L. Christopherson, "Speech identification difficulties of hearing-impaired elderly persons: the contributions of auditory processing deficits," Journal of Speech and Hearing Research, vol. 34, no. 3, pp. 686-693, 1991.

[13] L. J. Trainor and S. E. Trehub, "Aging and auditory temporal sequencing: ordering the elements of repeating tone patterns," Perception and Psychophysics, vol. 45, no. 5, pp. 417-426, 1989.

[14] J. C. Cooper and G. A. Gates, "Hearing in the elderly-the framingham cohort, 1983-1985: part II. Prevalence of central auditory processing disorders," Ear and Hearing, vol. 12, no. 5, pp. 304-311, 1991.

[15] J. P. Walton, H. Simon, R. D. Frisina, and P. Giraudet, "Agerelated alterations in the neural coding of envelope periodicities," Journal of Neurophysiology, vol. 88, no. 2, pp. 565-578, 2002.

[16] P. J. Price and H. J. Simon, "Perception of temporal differences in speech by "normal-hearing" adults: effects of age and intensity," Journal of the Acoustical Society of America, vol. 76, no. 2, pp. 405-410, 1984.

[17] S. Rosen, J. Walliker, J. A. Brimacombe, and B. J. Edgerton, "Prosodic and segmental aspects of speech perception with the house/3M single-channel implant," Journal of Speech and Hearing Research, vol. 32, no. 1, pp. 93-111, 1989.

[18] K. L. Bopp and P. Verhaeghen, "Aging and verbal memory span: a meta-analysis," Journals of Gerontology B, vol. 60, no. 5, pp. P223-P233, 2005.

[19] H. A. Slagter, A. Lutz, L. L. Greischar et al., "Mental training affects distribution of limited brain resources," PLoS Biology, vol. 5, no. 6, article el38, 2007.

[20] Y.-Y. Tang, Y. Ma, J. Wang et al., "Short-term meditation training improves attention and self-regulation," Proceedings of the National Academy of Sciences of the United States of America, vol. 104, no. 43, pp. 17152-17156, 2007.

[21] G. L. Xiong and P. M. Doraiswamy, "Does meditation enhance cognition and brain plasticity?" Annals of the New York Academy of Sciences, vol. 1172, pp. 63-69, 2009.

[22] A. Chiesa and A. Serretti, "A systematic review of neurobiological and clinical features of mindfulness meditations," Psychological Medicine, vol. 40, no. 8, pp. 1239-1252, 2010.

[23] S. W. Lazar, C. E. Kerr, R. H. Wasserman et al., "Meditation experience is associated with increased cortical thickness," NeuroReport, vol. 16, no. 17, pp. 1893-1897, 2005.

[24] E. B. Short, S. Kose, Q. Mu et al., "Regional brain activation during meditation shows time and practice effects: an exploratory FMRI study," Evidence-Based Complementary and Alternative Medicine, vol. 7, no. 1, pp. 121-127, 2010. 
[25] B. R. Cahn and J. Polich, "Meditation states and traits: EEG, ERP, and neuroimaging studies," Psychological Bulletin, vol. 132, no. 2 , pp. 180-211, 2006.

[26] E. B. Short, S. Kose, Q. Mu et al., "Regional brain activation during meditation shows time and practice effects: an exploratory FMRI study," Evidence-Based Complementary and Alternative Medicine, vol. 7, no. 1, pp. 121-127, 2010.

[27] N. Srinivasan and S. Baijal, "Concentrative meditation enhances preattentive processing: a mismatch negativity study," NeuroReport, vol. 18, no. 16, pp. 1709-1712, 2007.

[28] M. Grassi and A. Soranzo, "MLP: a MATLAB toolbox for rapid and reliable auditory threshold estimation," Behavior Research Methods, vol. 41, no. 1, pp. 20-28, 2009.

[29] D. M. Green, "A maximum-likelihood method for estimating thresholds in a yes-no task," Journal of the Acoustical Society of America, vol. 93, no. 4, pp. 2096-2105, 1993.

[30] D. M. Green, "Stimulus selection in adaptive psychophysical procedures," Journal of the Acoustical Society of America, vol. 87, no. 6, pp. 2662-2674, 1990.

[31] F. E. Musiek, A. G. Reeves, and J. A. Baran, "Release from central auditory competition in the split-brain patient," Neurology, vol. 35, no. 7, pp. 983-987, 1985.

[32] K. Nie, G. Stickney, and F.-G. Zeng, "Encoding frequency modulation to improve cochlear implant performance in noise," IEEE Transactions on Biomedical Engineering, vol. 52, no. 1, pp. 64-73, 2005.

[33] D. D. Greenwood, "A cochlear frequency-position function for several species-29 years later," Journal of the Acoustical Society of America, vol. 87, no. 6, pp. 2592-2605, 1990.

[34] F.-G. Zeng, K. Nie, S. Liu et al., "On the dichotomy in auditory perception between temporal envelope and fine structure cues (L)," Journal of the Acoustical Society of America, vol. 116, no. 3, pp. 1351-1354, 2004.

[35] G. A. Studebaker, "A "rationalized" arcsine transform," Journal of Speech and Hearing Research, vol. 28, no. 3, pp. 455-462, 1985.

[36] A. Parbery-Clark, D. L. Strait, S. Anderson, E. Hittner, and N. Kraus, "Musical experience and the aging auditory system: implications for cognitive abilities and hearing speech in noise," PLoS ONE, vol. 6, no. 5, Article ID e18082, 2011.

[37] A. Heinrich, B. A. Schneider, and F. I. M. Craik, "Investigating the influence of continuous babble on auditory short-term memory performance," Quarterly Journal of Experimental Psychology, vol. 61, no. 5, pp. 735-751, 2008.

[38] M. K. Pichora-Fuller and P. E. Souza, "Effects of aging on auditory processing of speech," International Journal of Audiology, vol. 42, supplement 2, pp. S11-S16, 2003.

[39] L. Demany and C. Semal, "The role of memory in auditory perception," in Auditory Perception of Sound Sources, W. A. Yost, A. N. Popper, and R. R. Fay, Eds., Springer, New, NY, USA, 2007.

[40] B. K. Hölzel, U. Ott, H. Hempel et al., "Differential engagement of anterior cingulate and adjacent medial frontal cortex in adept meditators and non-meditators," Neuroscience Letters, vol. 421, no. 1, pp. 16-21, 2007.

[41] E. R. Valentine and P. L. G. Sweet, "Meditation and attention: a comparison of the effects of concentrative versus mindfulness meditation on sustained attention," Mental Health, Religion and Culture, vol. 2, pp. 59-70, 1999.

[42] A. P. Jha, J. Krompinger, and M. J. Baime, "Mindfulness training modifies subsystems of attention," Cognitive, Affective and Behavioral Neuroscience, vol. 7, no. 2, pp. 109-119, 2007.
[43] P. C. M. Wong, J. X. Jin, G. M. Gunasekera, R. Abel, E. R. Lee, and S. Dhar, "Aging and cortical mechanisms of speech perception in noise," Neuropsychologia, vol. 47, no. 3, pp. 693703, 2009.

[44] S. M. Rao, A. R. Mayer, and D. L. Harrington, "The evolution of brain activation during temporal processing," Nature Neuroscience, vol. 4, no. 3, pp. 317-323, 2001.

[45] N. Kraus, D. L. Strait, and A. Parbery-Clark, "Cognitive factors shape brain networks for auditory skills: spotlight on auditory working memory," Annals of the New York Academy of Sciences, vol. 1252, no. 1, pp. 100-107, 2012. 

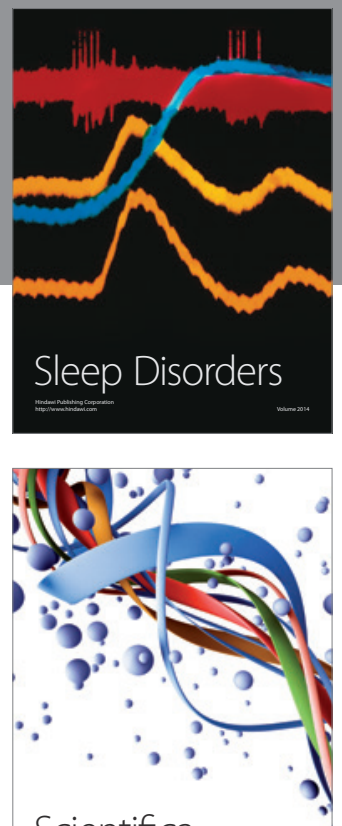

Scientifica
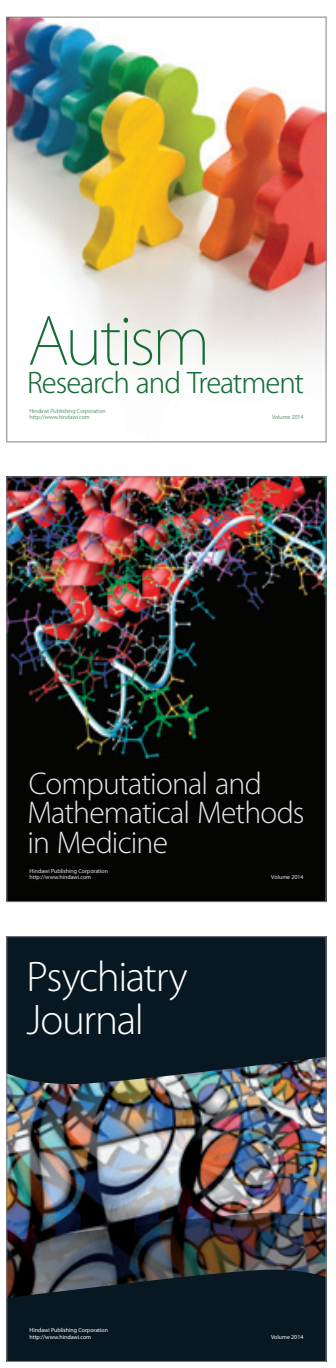
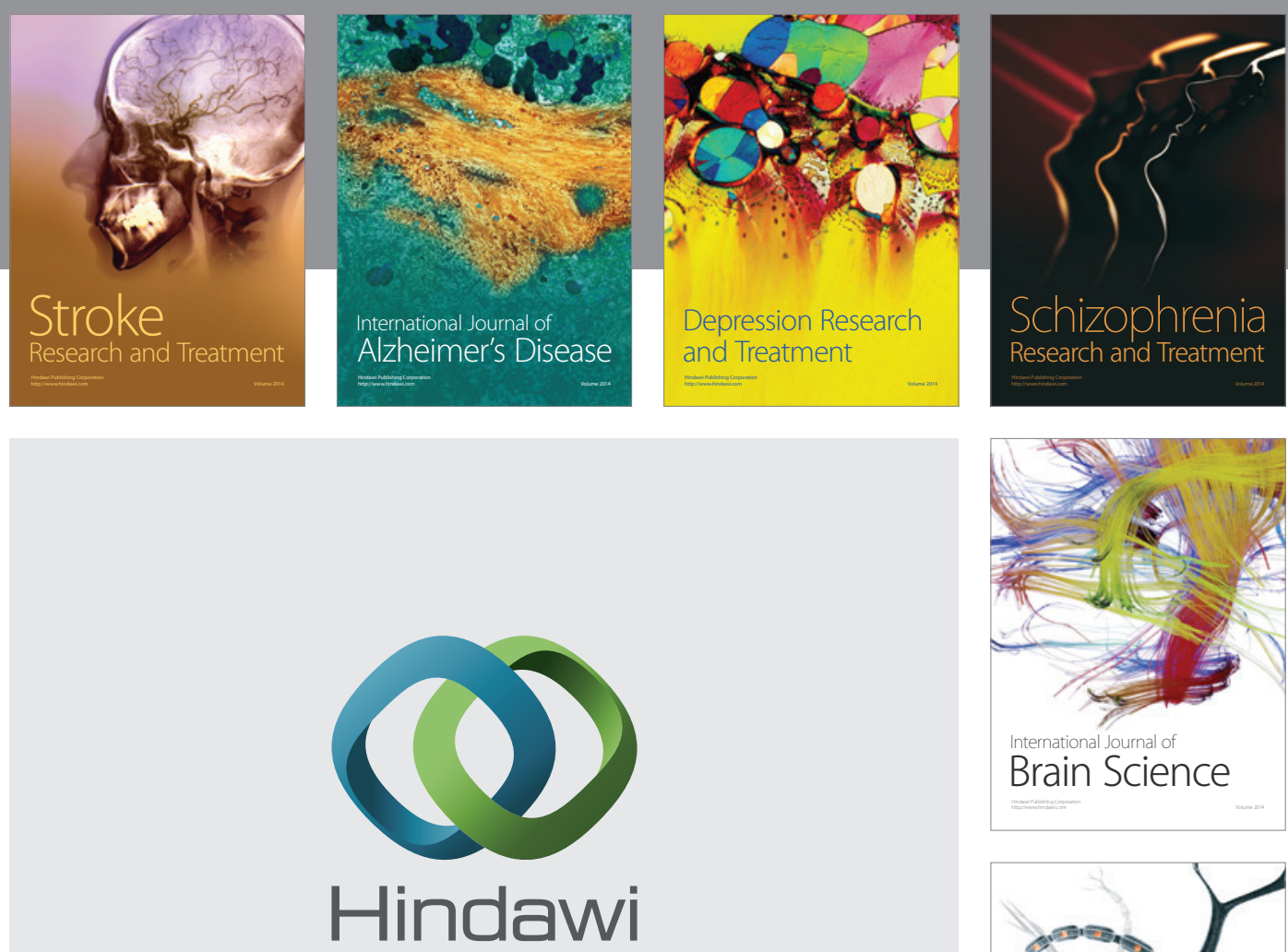

Submit your manuscripts at

http://www.hindawi.com
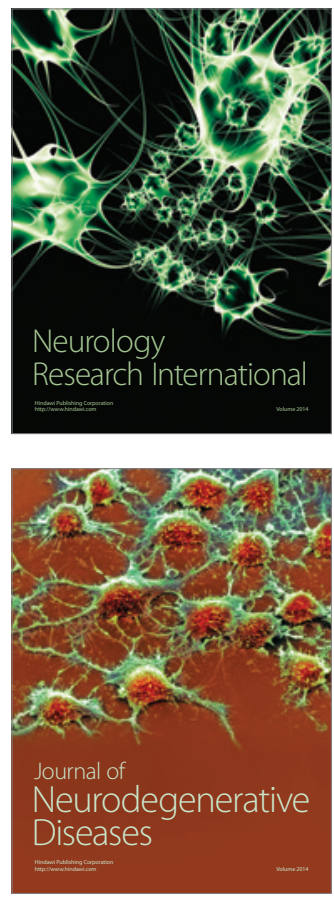

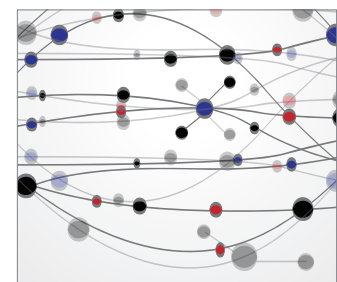

The Scientific World Journal
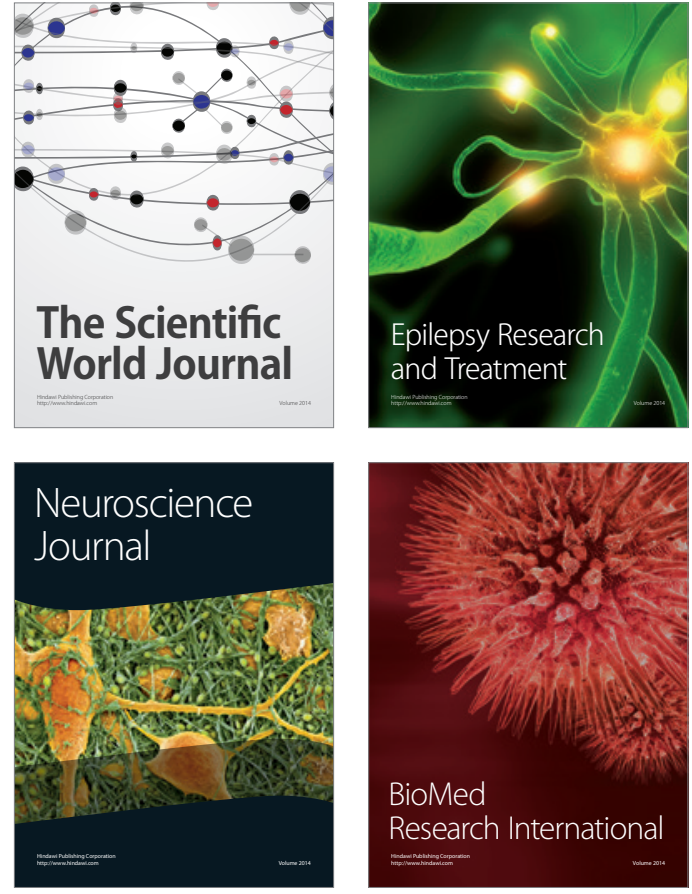

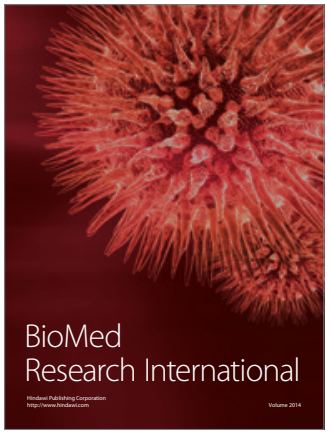

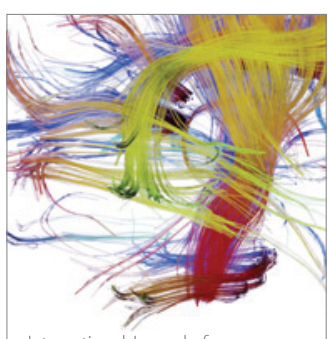

Brain Science

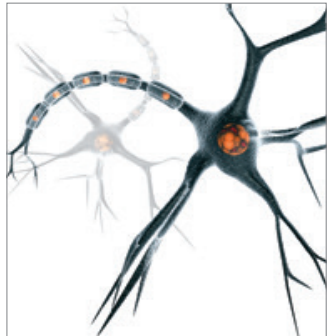

Neural Plasticity
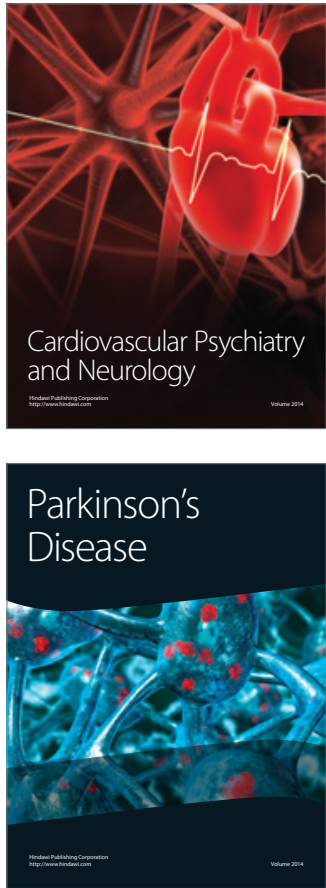